\title{
From Multi-User Virtual Environment to 3D Virtual Learning Environment
}

\author{
Daniel Livingstone ${ }^{\mathrm{a} *}$, Jeremy Kemp ${ }^{\mathrm{b}}$ and Edmund Edgar ${ }^{\mathrm{c}}$ \\ ${ }^{a}$ School of Computing, University of the West of Scotland, Paisley, UK; ${ }^{b}$ School of Library and \\ Information Sciences, San José State University, San José, USA; ${ }^{c}$ Social Minds Learning Systems, \\ London, UK
}

(Received 31 March 2008; final version received 8 September 2008)

\begin{abstract}
While digital virtual worlds have been used in education for a number of years, advances in the capabilities and spread of technology have fed a recent boom in interest in massively multi-user 3D virtual worlds for entertainment, and this in turn has led to a surge of interest in their educational applications. In this paper we briefly review the use of virtual worlds for education, from informal learning to formal instruction, and consider what is required to turn a virtual world from a Multi-User Virtual Environment into a fully fledged 3D Virtual Learning Environment (VLE). In this we focus on the development of Sloodle - a system which integrates the popular 3D virtual world of Second Life with the open-source VLE Moodle. Our intent is not simply to provide additional learning support features for Second Life, but to study more generally the ways in which integrated virtual environments can benefit teaching and learning, and this is the focus of our closing discussion.
\end{abstract}

Keywords: Multi-User Virtual Environments; Virtual Learning Environments; gamebased learning; Second Life; Immersive Virtual Worlds

\section{Introduction}

As testified by this special issue of $A L T-J$ dedicated to learning in Immersive Virtual Worlds (IVWs), there has been a recent marked growth of interest in the use of online graphical Multi-User Virtual Environments (also known as MUVEs) for educational purposes.

By and large, however, IVWs have not been designed or built specifically for teaching and learning, and many elements of support for learning and teaching which are now commonly found in web-based Virtual Learning Environments (VLEs) are completely absent. While it is eminently 9possible to use an existing VLE alongside a graphical IVW, we believe that integrating the two can help transform a Multi-User Virtual Environment into a true 3D Virtual Learning Environment - and this we have been working towards with the Sloodle project.

One part of Sloodle is a development project integrating the Second Life IVW and the Moodle VLE. In this paper we will outline the background for the application, how community input has been harnessed to drive the design to meet the needs of educators and outline findings from current work piloting Sloodle.

\footnotetext{
*Corresponding author. Email: daniel.livingstone@gmail.com
} 
The other part has a much broader goal - to explore ways in which the primarily textdriven web can be usefully integrated with rich, graphical 3D immersive environments. This more general work - potentially applicable to any combination of IVW and VLE - forms a key part of the discussion closing this paper.

But first some background detail is necessary. We will briefly review some of the pertinent history of virtual worlds in education, and consider the potential need and benefits of integration with web-based learning support.

\section{Background}

Online multi-user virtual worlds have been in existence since the late 1970s - initially based on text descriptions of the world and the actors within it (Achterbosch, Pierce, and Simmons 2007). With the evolution from the first Multi-User Dungeon (MUD) to later MUD ObjectOriented (MOOs) came the ability for users to extend and modify the virtual world. Over time, a number of educational projects were established using text-based virtual worlds to support student, teacher or workplace community development and knowledge sharing or for use in teaching subjects ranging from classics to computer science (c.f. Schlager, Schank, and Godard 1996; Churchill and Bly 1999; Slator, Hill, and Del Val 2004).

Up until recent years IVWs remained very much fringe activities for both entertainment and serious use, enjoyed or employed by a relatively small audience. The contemporary picture is somewhat different, with Massively Multi-Player Online Games (MMOGs) now commanding a user base in the tens of millions (Achterbosch, Pierce, and Simmons 2007) and interest in the use of IVWs for education similarly booming. Current Eduserv Foundation surveys have found that over half of UK higher education institutions now have some form of presence or activity in Second Life (Kirriemuir 2007). Yet few IVWs have been designed specifically to support educational objectives.

Consider the early use of the world-wide web in education as a corollary example at this point. Large scale adoption of web-based e-learning required the development of VLEs to support and simplify common management, instructional, learning, assessment and communication tasks used in e-learning (Cook 1999). Without environments purposefully developed for education, it is likely that many educators would find it more challenging to effectively employ the web and deliver e-learning.

What support for e-learning can be found in IVWs? In comparison with VLEs, IVWs provide strong support for synchronous interactions and collaborations, and immersive environments for experiential and constructionist learning (c.f. Dickey 2003; Livingstone and Kemp 2006; Mason and Moutahir 2006). On the other hand, IVWs provide poor support for asynchronous collaboration, where students are online at different times, and tend not to provide support for learning management, assessment or reflective practice (Kemp and Livingstone 2006). There are a few existing examples, however, of learning support being added to existing IVWs.

First, we consider two examples developed using the Active Worlds platform: the River City Project (Dede et al. 2004) and Quest Atlantis (Barab et al. 2005). Active Worlds is an IVW which has been available since 1995 and now looks somewhat dated. By default, Active Worlds splits the user interface into a number of areas, as seen in the image of the River City Project (Figure 1).

The view and action space contains a number of controls for the $3 \mathrm{D}$ camera and for the user's avatar, with the avatar and surrounding space shown in the second area. Below this is a chat window where typed chat messages to and from other avatars appear. The final area, although labelled 'Student Workspace', is in fact a web-browser built into the 


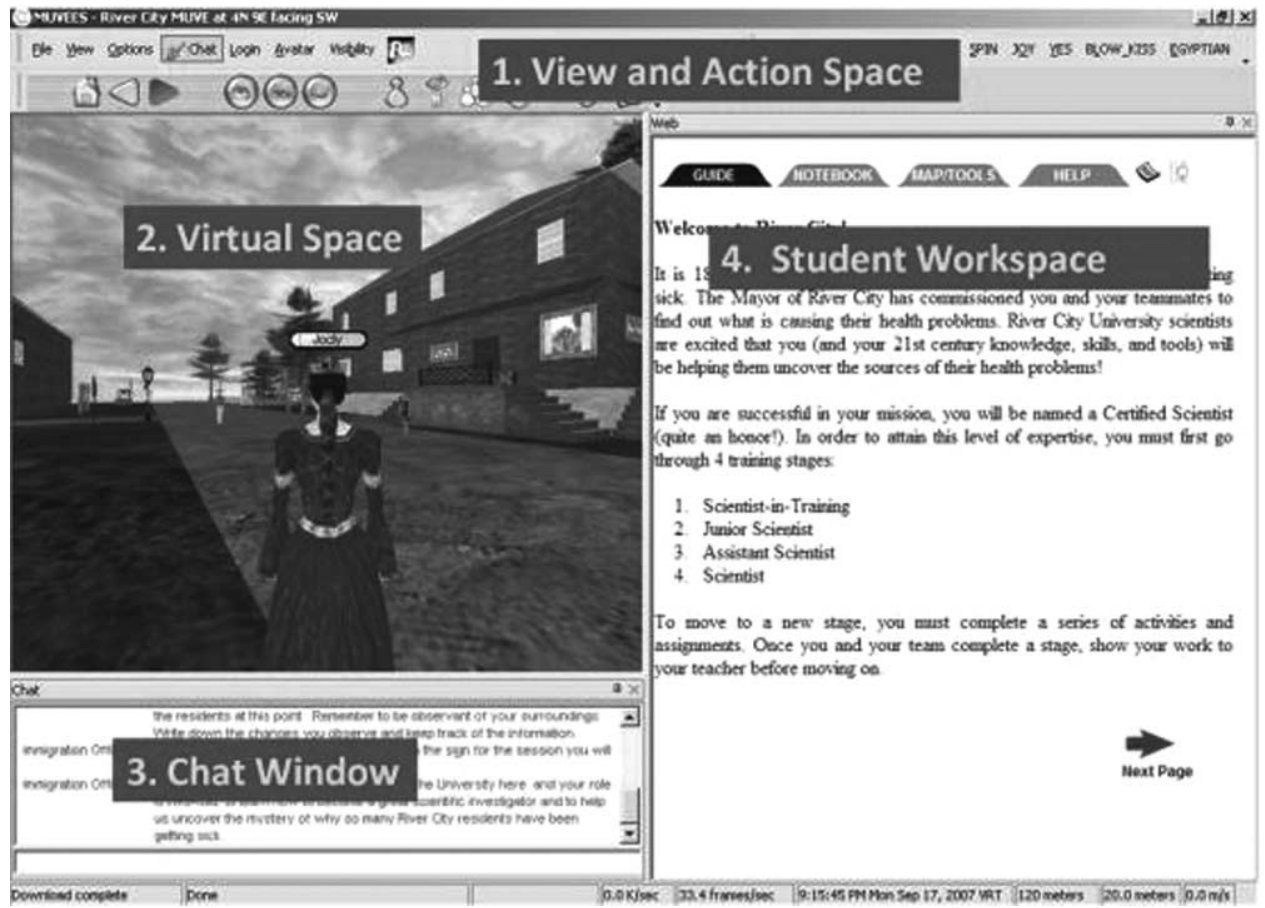

Figure 1. Labelled diagram showing the different interactive elements in the River City/Active Worlds user interface. The 'Student Workspace' appears in a web-browser window that is part of the standard Active Worlds UI. River City Project image used with permission and retrieved from http:/ /muve.gse.harvard.edu/rivercityproject/view/rc_views_interface.htm.

interface. As with other IVWs, Active Worlds was not developed as an e-learning platform; the developers of the River City Project added the learning support they felt was required via a separate website which is accessed through the built-in browser. The website provides a guide, a notebook, a map, tools and help to the students engaged in the virtual space.

More generally, the use of built-in guides and notebooks for structured notetaking was found by Laurillard et al. (2000) to support more effective use of CD-ROM e-learning resources by providing a learning narrative over student interactions. While the River City Project has its own story narrative, it is not too much of a stretch to see how similar use of guides and notebooks helps scaffold, structure and focus attention on the learning activities - and away from the mechanical activities involved in navigating and exploring a 3D space.

While visually very different, Quest Atlantis uses a similar technique, providing a custom-built interface in the web-browser window to support learners engaged in activities in that project. The Quest Atlantis support interface is designed to look more like a user interface from a computer game - with a prominent display of the number of collected 'lumin' crystal rewards and a number of colourful buttons to access information on current quests, friends lists, personal information and more. ${ }^{1}$

While both Quest Atlantis and the River City Project present good examples of integrating web support into 3D IVW learning environments, both are also examples of heavily customised virtual worlds, with teams of developers working to create the virtual worlds and develop highly specialised and bespoke web-based learning spaces to complement the 3D graphical environment. 
Second Life has proven popular with many instructors for its flexibility and the possibility of creating or customising teaching and learning for their own students - without reliance of teams of developers to create the learning content for them. Yet Second Life lacks effective built-in learning support and it is, perhaps, asking too much of educators to create such learning support mechanisms themselves.

\section{Sloodle}

The need for better support for e-learning in $3 \mathrm{D}$ virtual worlds is felt by many, and has appeared as an issue in a number of surveys, e.g.:

integration of Second Life (which is synchronous) with other Web 2.0 technologies (e.g. wiki, forum, etc) is critical. (Kirriemuir 2007, 20)

The core concept of Sloodle is to bring flexible support for teaching and learning to 3D virtual worlds via integration with a VLE - specifically Second Life and the open-source Moodle VLE. Second Life was selected as the IVW of choice because of its popularity, extensibility and the enthusiastic community of educators using the platform. The Moodle VLE was selected because of its open-source nature and again a sizeable online community supporting the platform.

In its earliest conception, Sloodle would provide a form of 3D 'virtual classroom', presenting access to a Moodle course in 3D form and automatically mirroring the weblayout of the course (Figure 2) (Kemp and Livingstone 2006). The different blocks making up a course would each have a 3D correlate for avatars to interact with. Thus a 3D calendar would highlight important dates; clicking on these would read out the event information to students or an assignment could be seen to move closer to the deadline by having a flag descend a pole as the due date approached. These designs were innovative though not informed by practical teaching trials in Second Life and with no input from potential users to determine actual requirements. They focused on the aesthetic properties of the Moodle layout structure and directly mapping user interface affordances.

An initial problem to be solved was how to identify the Second Life avatar belonging to a particular Moodle user. So, for example, when a student submits a blog directly from Second Life (see below), the entry needs to be added to Moodle with the student's Moodle

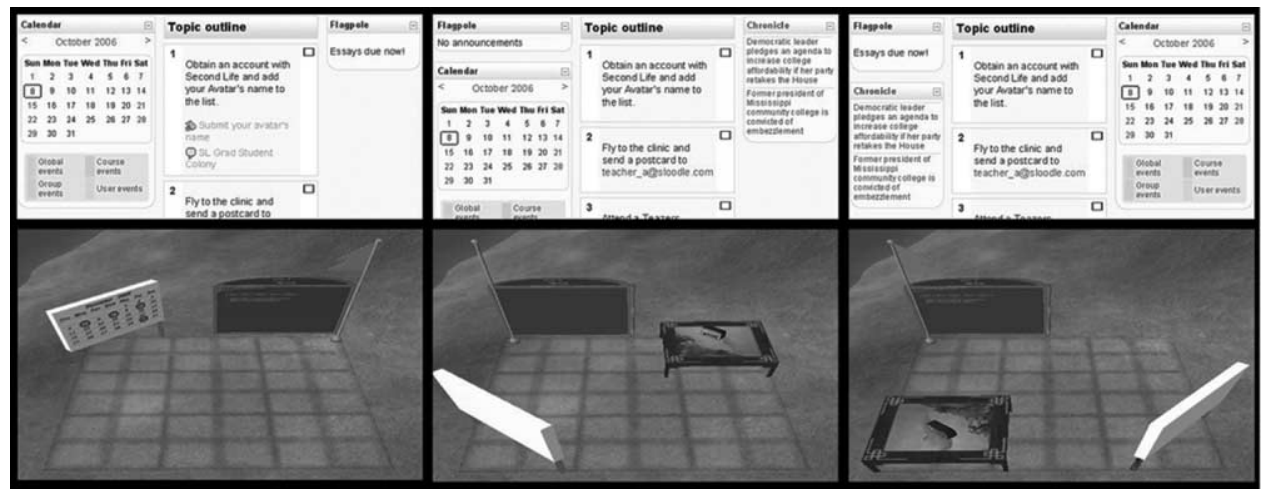

Figure 2. The Sloodle classroom - realising Moodle courses in 3D. 
user details. A number of different approaches and utilities have been tried for this - with a simple 'registration booth' being the preferred solution. This appears as a small 3D kiosk in Second Life. When a user clicks on this, they receive a prompt taking them to an avatar registration page on their institution's Moodle VLE. As they need to log into Moodle to access this page, it is then possible to create and store a link of their Second Life avatar details against their Moodle credentials.

This is implemented through a combination of new scripts running on the Moodle server and scripted objects built in Second Life. The former are developed in PHP Hypertext Processor (as is Moodle itself) and can be installed as any other optional module in Moodle. The latter are 3D models which incorporate scripts developed in the Second Life 'Linden Scripting Language' (LSL). Sloodle does not modify the Second Life client software itself in any way.

As an example of how Sloodle features are implemented, consider the Sloodle webintercom. This mirrors chat between Second Life and Moodle, allowing students to participate in chats from either environment and it also archives Second Life chat on the Moodle server. Thus, chat activities can be conducted using either Second Life or a regular webbrowser, with the Sloodle scripts and objects supporting the interaction in Second Life (Figure 3).

Over time, the direction that Sloodle has taken has changed somewhat from the original '3D classroom' concept. Rather than being constrained by the layout defined for the web, Sloodle now aims to provide tools which can be used in a variety of different spaces within the virtual world. Sloodle tools now include:

- Object distributors which allow objects to be passed to students via a web-interface in Moodle and which enable avatars belonging to registered students to select items inworld.

- A Second Life user-interface enhancement; a toolbar which enables blogging to a user's Moodle profile, adds animated classroom gestures to Second Life, and which

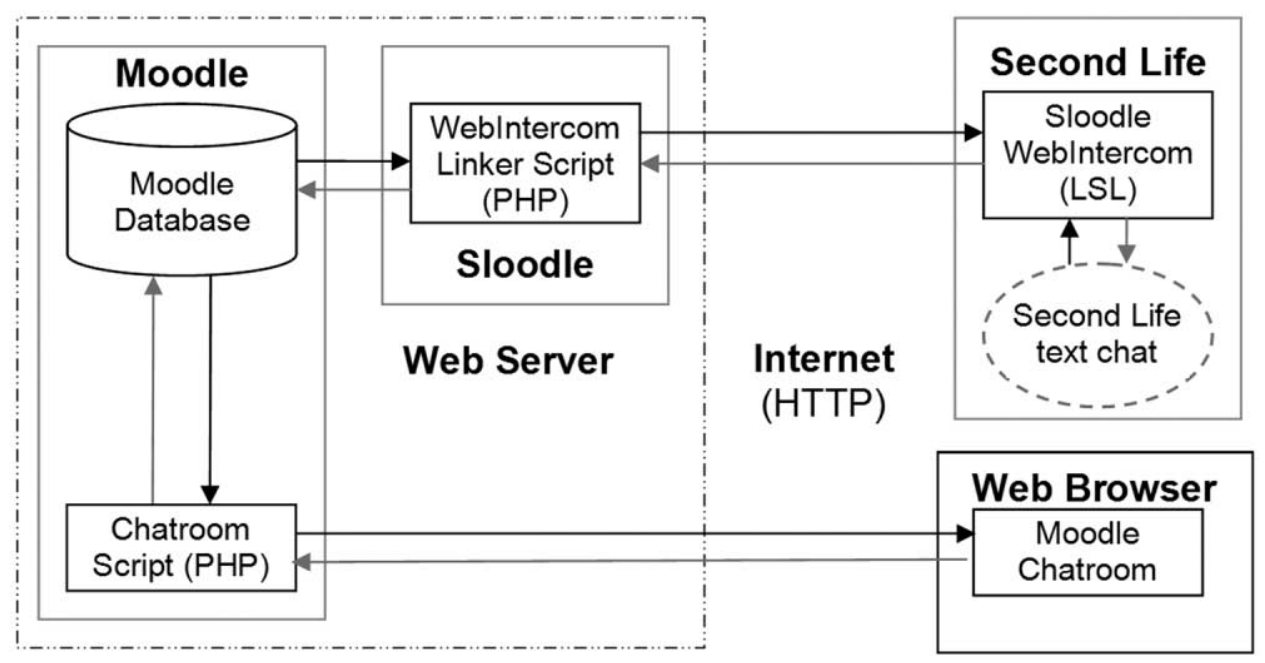

Figure 3. Sloodle is implemented using a mixture of PHP scripts residing on a Moodle server and LSL scripted objects in Second Life, as shown in this schematic of the web-intercom. Communication between the two is via HTTP, the same protocol used by standard web-browsers. 


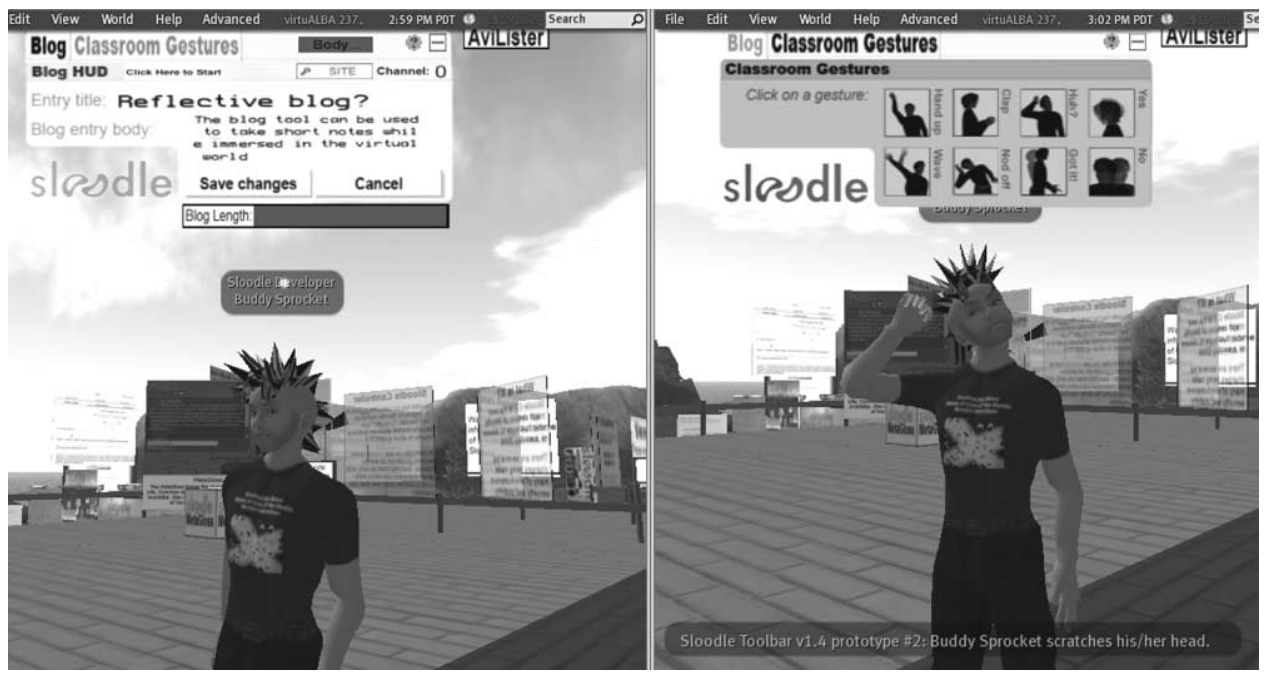

Figure 4. The Sloodle Toolbar acts as a user-interface extension in Second Life, allowing users to write directly to web-logs in Moodle. Additional features of this extensible tool include classroom animations and the ability to match avatars to their corresponding Moodle users.

allows a user to query Moodle for the Moodle identities (if any) of avatars nearby in the 3D virtual space (Figure 4).

- A Sloodle block which can be added to pages in Moodle. This shows a user's Second Life avatar identity and allows access to a number of user and administration tools in Moodle (Figure 5).

- A new Moodle assignment type and associated Second Life object for collecting assignments inside Second Life. This allows students to submit 3D projects created in Second Life to a virtual drop-box. Instructors can review submission details in Moodle, collect coursework from the drop-box in Second Life at their convenience and enter grades and provide feedback as normal in Moodle.

How these tools are used in practice is to some extent instructor and student dependent. Some features are aimed at supporting class and learning management, while others are aimed more at supporting the learning process itself. For example, using the web-intercom or the avatar listing feature of the toolbar, an instructor can record attendance in Second Life. Conversely, making chat sessions in Second Life available for review may be of benefit to students as an aide-memoir and to help them reflect later on their experiences in the virtual world.

Enabling blogging to Moodle from within Second Life can also be used as a tool to aid reflection and support learning - similar to the use of a notebook in the River City Project. Students can record notes while within the 3D environment, which are then recorded in Moodle along with URLs linking back to the location in the 3D world where the note was taken. These can be reviewed later by the student, may be extended if desired, and can also be viewed by classmates or instructors. These can be helpful in disseminating knowledge amongst classmates in a community of learners and could also be used for formative assessment of student learning.

In wanting to direct our efforts most usefully, we have attempted to involve as wide a community as possible in shaping the development of Sloodle. How we have strived to achieve this is outlined next. 


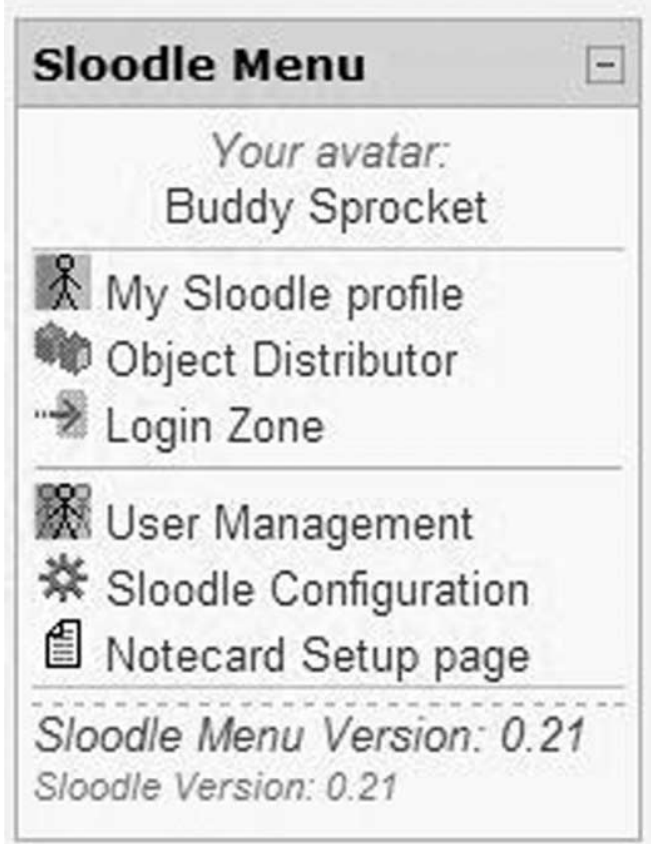

Figure 5. Sloodle menu block. A number of administrative features are available via Moodle - including the ability to send items to avatars inside Second Life without having to log in to the 3D environment.

\section{User-driven development}

Sloodle development began, towards the end of 2006, with the creation of a number of proof-of-concept demonstration elements within Second Life - those seen in Figure 2 and an early version of the web-intercom.

Almost immediately after a number of demonstration events were held in Second Life, a Moodle website was set up as the online home for the project, with forums and courses for developers and potential users. This was followed by an online survey to help gauge demand and refine Sloodle requirements. While the number of respondents was low, the feedback, combined with attendance and comments at demonstration events, satisfied us that there was some demand for Sloodle. Regular online project meetings and a number of online forums continue to provide avenues for gathering feedback and comments from the community.

Development work progressed and a second, more detailed, online survey was conducted in late 2007. One hundred and ninety-three responses to the call were received, with around 155 completing the survey. Questions asked participants to rate the perceived usefulness of a large range of possible Sloodle features with answers on a five-point scale from 'Unnecessary' to 'Vital'.

Strong demand was indicated in that almost every feature suggested was rated as being at least 'somewhat useful' by the majority of respondents. Indeed, many features were perceived by over $60 \%$ of respondents as being at least 'very useful'. The results of the survey validated many of the development decisions taken in the intervening year, and have 
highlighted priorities for future development work. There is limited space to review the results here, but fuller results may be viewed online (http://www.sloodle.org/mod/resource/ view.php?id=523).

Of the survey's five most highly rated possible features, only one had been prototyped prior to the survey - a Second Life drop-box. This has since been completed, as noted above. Three of the five are features that are not dependant on Moodle integration, these being: pre-prepared objects to support Second Life orientation; a ready to use 3D classroom; and an item to manage objects in a 3D virtual classroom. To some degree these are already available in Second Life and we have not allocated resources to developing these as yet. The single most highly rated feature was a Second Life collaborative whiteboard. There are a number of significant challenges in creating a collaborative sketching tool which can be used inside Second Life. For example, the client software does not easily allow detection of the position of the mouse as it moves over a surface - essential for drawing. An alternative approach is to create sketches using an external web-application and display the sketches in Second Life using the software client's ability to render images and web-pages. Following the survey, work on implementing a Sloodle collaborative whiteboard began - as detailed below.

Interestingly, the next three most highly rated features were all ones which were already working or in development - the web-intercom, blogging support and gestures.

\section{The Sloodle pilot}

During the development of Sloodle, small numbers of early adopters have been using the tools to support their teaching - including some of the Sloodle developers themselves. A more formal pilot was conducted during the first few months of 2008. Support was given to participants wishing to set up Sloodle for their own classes during the pilot, including the provision of a ready to use Moodle/Sloodle server. ${ }^{2}$

A dedicated course was set up to support the pilot at Sloodle.org, with details of the pilot and a prominent reminder that pilot activity may be used in research. As well as forums, regular facilitated discussions were held as a core part of the pilot activity - these being led by either members of the Sloodle development team or by pilot participants sharing their experience with others and inviting discussion. The topics for the meetings were all based on the experience of teaching and learning in virtual worlds. These were set up to allow participants to discuss the impact of various issues and the possible role of integrated VLEs in Second Life education activities. Some meetings were led by pilot participants themselves, allowing them to share their experiences of using Sloodle with their classes.

Live meetings in Second Life used the web-intercom to archive typed chat. Where voice communication was used, audio recordings were kept for later transcription. Individual rights to privacy and anonymity have been respected, although we are thankful to those participants willing and able to share their experiences more publicly, as in the example given below.

While the pilot evaluation is still in progress, early feedback has proven supportive and informative - revealing unexpected uses of existing tools as well as highlighting potential shortcomings and additional requirements. For example, problems trying to distribute items in Second Life to avatars who were not registered already on Moodle have led to changes and additions to the tools object distribution. An unexpected, and unresolved, issue is how to deal with Moodle users who have avatars on both Teen Second Life and the adult Second Life - and who would like both to be registered with the same Moodle account. 
A collaborative whiteboard was also trialled during the pilot. Feedback indicated that the drawing tool was not intuitive or easy to use, while alternative web-based sketch tools already exist. Instead of continuing with this, development has shifted into the creation of a collaborative web-browser to extend the very limited functionality available in Second Life for presenting web-content. Being able to share control of a virtual mouse and to use hyperlinks in web-pages are two notable extensions over the standard functionality - Second Life's normal HTML presentation being limited to a non-interactive image of what the webpage looks like.

\section{Innovative uses of existing features}

Perhaps the most promising feedback from any pilot is when users are making effective use of tools in ways unintended by the developers. The intended use of the Sloodle Second Life distribution boxes was to make it possible for instructors to pass out, or students to select for their avatars, the different Sloodle tools via a web-interface in Moodle. An alternative use found by one participant (Chris Surridge of KAIST, South Korea) for this was to fill a distribution box with avatar clothing, vehicles, furniture and toys - to allow students to kit out their avatars from a wide range of pre-selected 'goodies'. This in itself became a fun and engaging class activity for students, as they explored the items available and swapped and shared the things that they found. It also provided the language students with a context for practicing their English - to tell each other about the objects they had found.

The same participant has described how he has been able to motivate his language students by linking them with students in another country. The two groups of students have posed questions to each other using mp3 audio forums in Moodle (enabled by a Moodle plug-in), taken part in live video link-ups (again managed by a Moodle plug-in) and finally met and text-chatted in Second Life. The text-chat provides the ability for students to build upon their written language skills in a real-time conversational setting, and these have been recorded using the web-intercom.

A significant advantage of the web-intercom was the ability for students to review discussions at a later time - to examine passages that confused them during the initial discussion, or to look more closely at their own performance. Using the intercom, the chat activities were able to combine conversational use of English with enhanced support for reflection. As can be seen in Figure 6, the web-intercom lists both Second Life avatar and Moodle user names next to the recorded chat messages. In this example, this provided additional support as the students had first made contact with each other using their real names, before adopting pseudonyms within the virtual world.

The potential benefits for language learners were not considered during the development of the web-intercom, and this example highlights the importance of having educators and students adapt the use of tools to support their particular mix of class activities.

A final additional benefit experienced by the KAIST class was seen in having all learning activities supported by a common VLE, whatever the medium of communication. Thus, with Sloodle acting as a support, Moodle became the glue which held a wide range of activities together.

\section{Discussion}

The response to surveys, feedback from the pilot and general participation in forums at Sloodle.org all demonstrate that respondents perceive a real demand and need for improved 

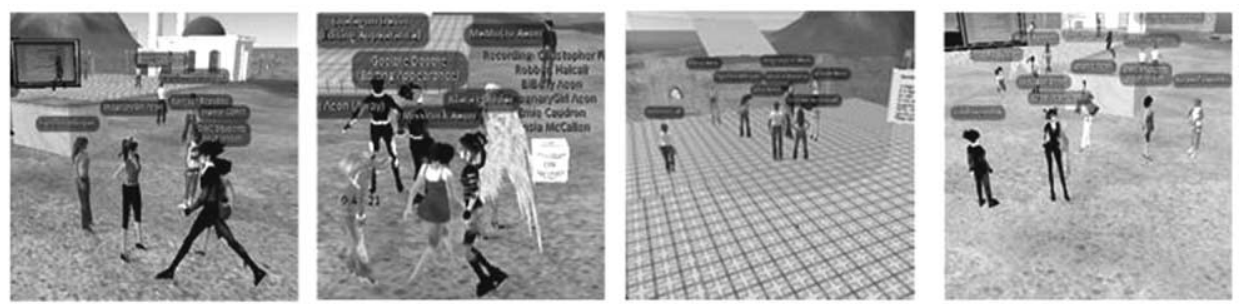

ep $>$ DKIVE $>$ Chats $\gg$ Second Life Chat Room $>$ Chat sessions

Wednesday, 12 March 2008, 01:39 PM --> Wednesday, 12 March 2008, 02:12 PM

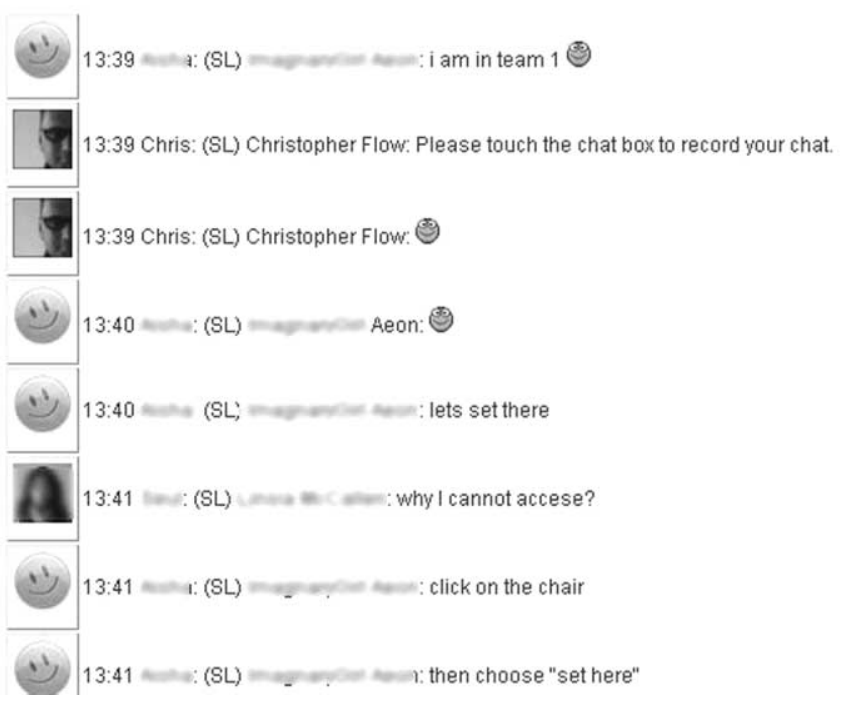

Figure 6. A Second Life chat archive in Moodle supports reflection on and review of class activities - and links Moodle and Second Life identities of participants.

integration of web-based learning support and Second Life. Although the software is still in the early stages of development, a community of educators interested in and using Sloodle to enhance teaching and learning in Second Life is emerging. We are now collecting and evaluating evidence which highlights how Sloodle might be effective in supporting learning and teaching in Second Life - and which is helping to drive the ongoing development of Sloodle itself.

Earlier, in the background review, we saw that effective use of e-learning technologies, including virtual worlds, requires that learners focus on learning, not on technology. This focus can be provided by a learning 'narrative', which provides goals and a means of assessing progress towards achieving those goals. This can form scaffolding for learning, but currently there is very limited support in Second Life itself for providing this.

Allowing virtual world activities to be initiated from Moodle, and having notes and discussions from those activities archived and stored back in Moodle, means instructors are able to support the development of just such a narrative within Moodle.

However, we have also found that the provisioning of educational tools is not a one-way process. It is educators themselves who have the greatest awareness of the most effective context in which to build an educational narrative appropriate to their desired learning 
outcomes and teaching styles. Tools have been used in ways not originally conceived by their designers, and observation of educators' innovations is feeding back, in an iterative process, into the design of the tools. As the pilot experience shows, asking educators which tools they imagine would be useful for learning and teaching is not sufficient. We need to provide prototype tools and learn from how they are used in practice.

The ability to view web-pages from within Second Life seems to present new possibilities, yet normal use of VLEs is predicated on restricting access to individuals with appropriate permissions. This leaves a number of issues to consider if content from Moodle is to be displayed inside public spaces in an IVW such as Second Life. While the collaborative web-browser allows groups of users to share a $3 \mathrm{D}$ discussion space in the IVW while browsing websites together, questions remain over how this could or should be used with Moodle specifically.

\section{Conclusions}

As IVWs become more commonly used for education and learning, the issues of providing effective support for teaching and learning are likely to recur across other platforms and environments.

While it is possible to use discrete and separate tools for such support, greater integration is required to realise the full potential of the current generation of $2 \mathrm{D}$ and $3 \mathrm{D}$ learning environments. This is beyond the resources of individual educators working with IVWs. We have attempted to address this for one of the most popular IVWs with the creation of Sloodle, an open-source project to integrate Second Life and Moodle.

We have found strong evidence of demand for tools such as Sloodle and, through the ongoing pilot, have also found evidence that Sloodle is already proving useful to a number of educators. We are continuing to gather feedback and ideas from educators to help drive the creation of additional tools to support learning, and hope that this will allow Sloodle to grow in usefulness to an increasingly wide audience of educators.

Like Moodle, all software used in Sloodle may be freely copied, altered and redistributed, leveraging the underlying strengths of both the Open Source development model and the Second Life platform. Educators and learning technologists may modify and customise the Sloodle environment to suit personal and institutional preferences.

The evolution from bespoke (and often expensive and proprietary) learning games to bottom-up, participatory systems mirrors the evolution seen elsewhere in the web, expressed in the growth of blogs and social networking systems (Jenkins 2006). It is our intention that Sloodle will support this transition, making it easier for IVWs to become fully integrated components of institutional e-learning systems and strategies.

\section{Acknowledgements}

Sloodle is supported and funded by the Eduserv Foundation. Early development of Sloodle was supported by The Carnegie Trust for the Universities of Scotland. Thanks to the editors and anonymous reviewers for their perceptive comments. Thanks also to Bronwen for her careful reading of the final draft.

\section{Notes}

1. See http://atlantis.crlt.indiana.edu/ for images of the Quest Atlantis interface.

2. http://moodlefarm.socialminds.jp/. Moodlefarm now hosts 340 Moodle/Sloodle systems, with 250 different users. The majority of these are not associated with the current Sloodle pilot. 


\section{References}

Achterbosch, L., R. Pierce, and G. Simmons. 2007. Massively multiplayer online role-playing games: The past, present, and future. Computers In Entertainment 5, no. 4: article no. 9.

Barab, S.A., M. Thomas, T. Dodge, R. Carteaux, and H. Tuzun. 2005. Making learning fun: Quest Atlantis, a game without guns. Educational Technology Research and Development 53, no. 1: 86-107.

Churchill, E.F., and S. Bly. 1999. Virtual environments at work: Ongoing use of MUDs in the workplace. In Proceedings of the international joint conference on work activities coordination and collaboration. San Francisco: ACM.

Cook, J. 1999. Virtual Learning Environments: Making the Web easy to use for teachers and learners. University of Bristol: LTSS.

Dede, C., B. Nelson, D.J. Ketelhut, and C. Bowman. 2004. Design-based research strategies for studying situated learning in a Multi-User Virtual Environment. Paper presented at the 6th International Conference on Learning Sciences, 22-26 June, in Santa Monica, California.

Dickey, M.D. 2003. Teaching in 3D: Pedagogical affordances and constraints of 3D virtual worlds for synchronous distance learning. Distance Education 24, no. 1: 105-21.

Jenkins, H. 2006. Confronting the challenges of participatory culture: Media education for the 21st century. Chicago: MacArthur Foundation.

Kemp, J., and D. Livingstone. 2006. Putting a Second Life 'Metaverse' skin on learning management systems. Paper presented at the Second Life Education Workshop, 20 August, at SLCC, San Francisco.

Kirriemuir, J. 2007. An update of the July 2007 'snapshot' of UK higher and further education developments in Second Life. Bath: Eduserv Foundation.

Laurillard, D., M. Stratfold, R. Luckin, L. Plowman, and J. Taylor. 2000. Affordances for learning in a non-linear narrative medium. Journal of Interactive Media in Education, no. 2. http:// www.jime.open.ac.uk/00/2

Livingstone, D., and J. Kemp. 2006. Massively multi-learner: Recent advances in 3D social environments. Computing and Information Systems Journal 10, no. 2, School of Computing, University of Paisley.

Mason, H., and M. Moutahir. 2006. Multidisciplinary experiential education in econd Life: A global approach. Paper presented at the Second Life Education Workshop, 20 August, at SLCC, San Francisco.

Schlager, M., P. Schank, and R. Godard. 1996. Tapped in to teacher professional development: An on-line conference center for education communities. Paper presented at the Serious Uses of MUDs workshop, 16-20 November, at CSCW '96, Boston, MA.

Slator, B.M., C. Hill, and D. Del Val. 2004. Teaching computer science with virtual worlds. IEEE Transactions on Education 47, no. 2: 269-75. 